\title{
Perfil dos atendimentos no serviço de Periodontia da Faculdade de Odontologia da UFRJ
}

\author{
Cláudia Callegaro de Menezes*; Raphaelle Emmanuelle Almeida Oliveira**; German Villoria***; Maria \\ Cynésia Medeiros de Barros $* * * *$
}
* Doutoranda, Programa de Pós-graduação em Odontologia, área de concentração Periodontia, Faculdade de Odontologia da UFRJ
** Cirurgiã-dentista graduada pela UFRJ
*** Cirurgião-dentista, Departamento de Clínica Odontológica, área de Periodontia, Faculdade de Odontologia da UFRJ
**** Professora Associada, Departamento de Clínica Odontológica, área de Periodontia, Faculdade de Odontologia da UFRJ

Recebido em 01/04/2019. Aprovado em 24/10/2019.

\begin{abstract}
RESUMO
O estudo teve como objetivo conhecer o perfil do serviço de atendimento prestado pela disciplina de Periodontia II da Faculdade de Odontologia da Universidade Federal do Rio de Janeiro, por meio de levantamento de dados dos atendimentos realizados em um período de 10 anos (2007 a 2016). Foram analisados o protocolo de atendimento, número total de procedimentos realizados por semestre e por estudante de graduação, número de exames periodontais, profilaxias, instruções de higiene oral, procedimentos de raspagem supragengivais, procedimentos de raspagem subgengival e alisamento radicular realizados por semestre. As informações relacionadas ao perfil dos usuários foram coletadas a partir do prontuário e incluíram dados sociodemográficos, diagnóstico das condições periodontais e condições sistêmicas associadas. No período estudado foram realizados 13.810 procedimentos clínicos, em média 690,5 por semestre. Em amostragem de 715 usuários a idade variou entre 12 e 85 anos, sendo a faixa etária predominante entre 45 e 64 anos, equivalente a 55,38\% da amostra. Na amostra, 14,55\% foram diagnosticados com gengivite e $85,45 \%$ com periodontite, sendo $93,94 \%$ dos casos periodontite crônica e $6,06 \%$ periodontite agressiva. Foram identificadas condições sistêmicas de saúde associadas aos quadros de doença periodontal. As condições mais relevantes informadas pelos pacientes foram hipertensão arterial $(25,87 \%)$, doenças respiratórias como sinusite e rinite alérgica $(13,29 \%)$, diabetes mellitus $(10,35 \%)$ e tabagismo $(7,70 \%)$. Foi possível conhecer em detalhes o funcionamento da clínica da disciplina de Periodontia II da FO/UFRJ e os resultados foram utilizados como base para melhorias no serviço. O perfil de saúde apresentado pelos usuários do serviço apontou para a necessidade de abordagem interprofissional.
\end{abstract}

Descritores: Administração de Serviços de Saúde. Periodontia. Ensino Odontológico. Cuidados Interprofissionais. 


\section{INTRODUÇÃO}

A avaliação da qualidade de serviços é parte integrante de planejamento e criação de estratégias para melhoria dos mesmos, sendo fundamental para a sua gestão. O levantamento de dados deve auxiliar na identificação de problemas e na organização de ações e serviços, avaliar a introdução de novas rotinas profissionais e mensurar o impacto das ações implementadas pelos serviços ${ }^{1-3}$.

$\mathrm{O}$ processo de avaliação da qualidade em serviço de saúde envolve tanto quem o utiliza como quem o produz, pois usuário e prestador ocupam posições diferentes no processo, embora ambos contribuam para o adequado funcionamento do serviço ${ }^{4,5}$. Torna-se essencial descrever os serviços que estão sendo prestados, visando analisar as diferentes percepções para, posteriormente, intervir buscando a melhoria contínua. A relação entre resolutividade e recursos humanos possibilita avaliar a qualidade do cuidado em saúde bucal, contribuindo para reorganização dos serviços de saúde.

Donabedian (1990) ${ }^{4}$ apresentou um conceito fundamental para o entendimento da qualidade em saúde, apontando sete atributos essenciais: eficácia, efetividade, eficiência, otimização, aceitabilidade, legitimidade e equidade. Estes pilares passaram a servir de norte para a avaliação dos serviços em saúde. Nesta ótica, são consideradas a percepção do paciente, porém amplia também a visão do serviço como um todo, considerando a efetividade, eficiência e otimização.

O Curso de Odontologia da Universidade Federal do Rio de Janeiro (UFRJ) é histórico no cenário da Odontologia Brasileira, com mais de 134 anos de existência. Foi criado a partir do Decreto $\mathrm{n}^{\circ}$. 9.311 em 25 de outubro de 1884 como um anexo à Faculdade de Medicina. A criação da Faculdade de Odontologia (FO) como unidade autônoma ocorreu em 28 de novembro de 1933, segundo o Decreto $\mathrm{n}^{\circ} .23 .512^{6}$ com sede própria no Campus da Praia Vermelha. Em 1973, o ciclo básico foi transferido para a Cidade Universitária na Ilha do Fundão, passando a ser ministrado no Centro de Ciências da Saúde

A missão da FO/UFRJ é promover a formação de recursos humanos na área de Odontologia por meio de ensino, pesquisa e extensão de qualidade, pautada em consistente valor científico orientado para a promoção da saúde, a prevenção e o tratamento das doenças bucais, incluindo a reabilitação da função e da estética oral e facial, respeitando a ética, a moral, as diferenças étnicas e de religião, em conformidade com as Diretrizes Curriculares Nacionais dos cursos de graduação em Odontologia ${ }^{7}$.

O serviço de Periodontia da FO/UFRJ recebe pacientes de qualquer região do município do Rio de Janeiro. Entretanto, por proximidade, a população mais atendida é oriunda da Área de Planejamento AP 3.1, na qual está localizado o curso. Segundo dados do IBGE, em 2012 o número de habitantes dessa área era de 896.350 , aproximadamente $14 \%$ do total de habitantes do município do Rio de Janeiro ${ }^{8}$.

O serviço odontológico na especialidade de Periodontia oferece atendimento odontológico à comunidade, incluindo procedimentos clínicos e cirúrgicos, realização de exames complementares por imagens (radiografia convencional, digital e tomografia). $\mathrm{O}$ atendimento envolve realizar diagnóstico, traçar o plano de tratamento individualizado para o usuário do serviço e executar tratamentos de baixa e moderada complexidade.

O protocolo de atendimento usado na Clínica de Periodontia II tem intuito de nortear o atendimento ao usuário, padronizando os procedimentos e facilitando a realização do diagnóstico e o planejamento correto do tratamento. Esse protocolo compreende sete etapas, que vão 
desde o primeiro contato com o paciente, até sua alta; sendo elas: (1) anamnese, (2) exame clínico periodontal, (3) exame radiográfico, (4) definição de diagnóstico periodontal, (5) plano de tratamento, (6) reavaliação e (7) manutenção ${ }^{9-11}$.

Além do atendimento clínico, a disciplina realiza atividades de extensão com ações de prevenção e promoção de saúde, as quais compõem o Projeto de Extensão "Reorientação da formação profissional em Odontologia - UFRJ - experiências no SUS com ênfase nos determinantes sociais de saúde e fatores de risco para as doenças periodontais". O projeto acontece desde 2012 e já obteve a participação de 423 estudantes de Odontologia entre os semestres de 2012.2 e 2018.2. Nestes 6 anos de existência já produziu 65 materiais educativos para melhorar o conhecimento dos usuários do serviço sobre como cuidar melhor de sua boca e as relações entre condições sistêmicas e saúde bucal. Dentre eles folders e painéis sobre dieta saudável e cuidados com a higiene na promoção de saúde bucal, instrução para os cuidados bucais, tabagismo e doença periodontal, tabagismo e seus efeitos na saúde bucal, vírus do papiloma humano, hipersensibilidade dentinária, diabetes mellitus e doença periodontal.

O objetivo deste estudo foi conhecer perfil do serviço de atendimento clínico odontológico prestado pela disciplina de Periodontia II da FO/UFRJ, por meio de levantamento de dados dos atendimentos realizados em um período de 10 anos (2007 a 2016).

\section{METODOLOGIA}

Trata-se de estudo descritivo com coleta de dados de prontuários. Foram analisados $\mathrm{o}$ protocolo de atendimento, número total de procedimentos realizados por semestre e por estudante de graduação, número de exames periodontais, profilaxias, instruções de higiene oral, procedimentos de raspagem supragengivais, procedimentos de raspagem subgengival e alisamento radicular realizados por semestre.

O Curso de Odontologia da UFRJ é um curso em tempo integral que atualmente tem duração de 4 anos. O serviço de atendimento ao público foco deste estudo é referente à disciplina de Periodontia II, com carga horária total de 60 horas por semestre, sendo 15 horas de conteúdo teórico e 45 horas de prática, as quais compreendem atendimento clínico na instituição e atividades de extensão extramuros em unidades da Estratégia Saúde da Família.

As informações relacionadas ao perfil dos usuários foram coletadas a partir do prontuário $\mathrm{e}$ incluíram dados sociodemográficos, diagnóstico das condições periodontais e condições sistêmicas associadas. Foi utilizada a Classificação das Doenças Periodontais da Academia Americana de Periodontia $^{12}$ para definição das doenças periodontais.

A pesquisa foi aprovada pelo CEP/ HUCFF/UFRJ sob o parecer de n 2.342.454.

\section{RESULTADOS}

No período estudado foram realizados 13.810 procedimentos clínicos no serviço de Periodontia da disciplina de Periodontia II, em média 690,5 por semestre, variando de 345 procedimentos no $1^{\circ}$ semestre de 2017 a 1036 no $2^{\circ}$ semestre de 2011 (gráfico 1).

As características demográficas da amostra dos usuários estão apresentadas na tabela 1 . Em amostragem de 715 usuários a idade variou entre 12 e 85 anos, sendo a faixa etária predominante entre 45 e 64 anos, equivalente a $55,38 \%$ da amostra.

As características do atendimento no serviço 
de Periodontia estão apresentadas na tabela 2. Os resultados referentes ao diagnóstico periodontal baseados na Classificação das Doenças Periodontais da Academia Americana de Periodontia ${ }^{12}$ estão apresentados na tabela 3.

Foram identificadas condições sistêmicas de saúde associadas aos quadros de doença periodontal, sendo as mais prevalentes nesta população apresentadas na tabela 4 . As condições mais relevantes informadas pelos pacientes foram hipertensão arterial (25,87\%), doenças respiratórias como sinusite e rinite alérgica $(13,29 \%)$, diabetes mellitus $(10,35 \%)$ e tabagismo $(7,70 \%)$.

Gráfico 1. Número de procedimentos clínicos por semestre

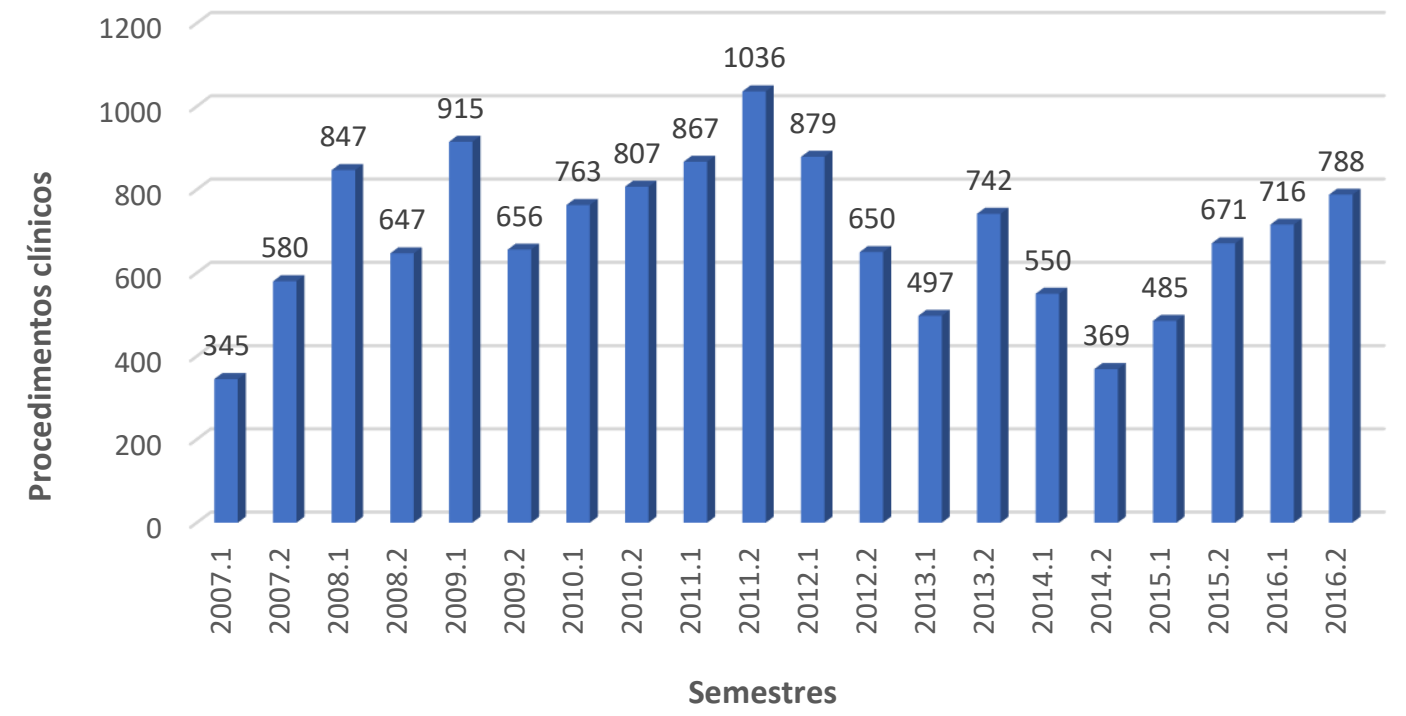

Tabela 1. Características demográficas dos usuários

\begin{tabular}{|c|c|c|}
\hline Dados demográficos & $\mathbf{N}$ & $\%$ \\
\hline \multicolumn{3}{|l|}{ Faixa etária } \\
\hline 12-14 anos & 5 & 0,69 \\
\hline 15-19 anos & 9 & 1,26 \\
\hline 20-34 anos & 73 & 10,21 \\
\hline $35-44$ anos & 145 & 20,78 \\
\hline 45-64 anos & 396 & 55,38 \\
\hline $65-85$ anos & 87 & 12,17 \\
\hline \multicolumn{3}{|l|}{ Sexo } \\
\hline Feminino & 445 & 62,24 \\
\hline Masculino & 270 & 37,76 \\
\hline
\end{tabular}


Tabela 2. Características do serviço de Periodontia

\begin{tabular}{lc}
\hline Variáveis (por semestre) & Média (DP) \\
\hline Número de estudantes & $31,85( \pm 7,19)$ \\
Número de procedimentos clínicos realizados & $21,7( \pm 2,61)$ \\
Número de exames clínicos periodontais & $75,1( \pm 18,98)$ \\
Número de profilaxias realizadas & $313,1( \pm 91,98)$ \\
Número de instruções de higiene oral & $74,9( \pm 18,86)$ \\
Número de procedimentos de raspagem supragengival & $106,45( \pm 29,73)$ \\
Número de procedimentos de raspagem subgengival & $132,4( \pm 47,85)$ \\
\hline
\end{tabular}

Tabela 3. Diagnóstico periodontal da amostra de usuários utilizando a Classificação das Doenças Periodontais da Academia Americana de Periodontia (AAP, 1999) $)^{12}$

\begin{tabular}{lcc}
\hline Diagnóstico periodontal & $\boldsymbol{n = 7 1 5}$ & $\mathbf{\%}$ \\
\hline Gengivite & 104 & 14,55 \\
Periodontite & 661 & 85,45 \\
$\quad$ Crônica & 574 & 93,94 \\
Agressiva & 37 & 6,06 \\
\hline
\end{tabular}

Tabela 4. Condições sistêmicas e tabagismo dos usuários da clínica de Periodontia II (2007-2016)

\begin{tabular}{lcc}
\hline Condição sistêmica e hábitos & $\mathbf{N}$ & $\mathbf{\%}$ \\
\hline Hipertensão & 185 & 25,87 \\
Outra doença cardiovascular & 58 & 8,11 \\
Diabetes & 74 & 10,35 \\
Doença respiratória & 95 & 13,29 \\
Depressão & 22 & 3,08 \\
Epilepsia & 12 & 1,68 \\
Hepatite C & 8 & 1,12 \\
Câncer & 19 & 2,66 \\
HIV & 3 & 0,42 \\
Estresse & 7 & 0,98 \\
Febre Reumática & 10 & 1,39 \\
Lúpus & 2 & 0,28 \\
Glaucoma & 8 & 1,12 \\
Hipertireoidismo & 7 & 0,98 \\
Hipotireoidismo & 18 & 2,52 \\
Anemia & 7 & 0,98 \\
Reumatismo & 11 & 1,54 \\
Gastrite & 5 & 0,70 \\
Pênfigo & 1 & 0,14 \\
Hepatite B & 3 & 0,42 \\
Osteoporose & 9 & 1,26 \\
Psoríase & 3 & 0,42 \\
Tabagismo & 55 & 7,70 \\
\hline
\end{tabular}




\section{DISCUSSÃO}

É crescente a necessidade de avaliação e monitoramento dos resultados alcançados em relação à organização e provisão dos serviços, incluindo os possíveis impactos produzidos na saúde e bem-estar das populações. "As ações individuais de saúde necessitam incluir todas as perspectivas do processo saúde-doença, ou seja, dar respostas de promoção e proteção à saúde, ações de recuperação e reabilitação" "13, sendo construídas com a participação dos diferentes sujeitos trabalhadores da saúde. As avaliações só terão legitimidade se houver compreensão e participação do usuário.

Baseando-se em Merhy $(1997,2002)^{14,15}$ e Campos $(2000,2003)^{16,17}$, compreende-se que os serviços de saúde precisam, necessariamente, situar suas práticas tendo o usuário como centro do processo terapêutico. Para tanto, os referidos autores apresentam dispositivos - acolhimento, vínculo, autonomia, responsabilização e resolubilidade - que juntos potencializam o processo de trabalho em saúde e, paralelamente, contribuem para a construção do cuidado, tendo como matriz a integralidade. Apesar desse estudo não ter focado na percepção do usuário, muitos dos elementos apresentados visam oferecer-lhe atendimento digno, de qualidade e que aborde ações educativas para o processo de melhoria da sua saúde geral e bucal.

O cuidado em saúde deve começar durante $o$ primeiro contato do paciente com o cirurgiãodentista - no caso o estudante de Odontologia em treinamento - para a sua recepção. $\mathrm{O}$ ambiente deve compreender organização, higiene, estética, conforto, sinalização e informativos sobre cuidados em saúde bucal. A utilização de um protocolo bem definido baseado em evidência científica traz ao serviço a organização necessária para o oferecimento de assistência ao usuário ${ }^{10,11}$. O serviço avaliado no estudo apresenta um protocolo de atendimento bem definido, com etapas sequenciais e baseado em achados científicos pertinentes ${ }^{9,18-21}$.

Outro aspecto importante, no que tange à qualidade do cuidado em saúde e à humanização do serviço, é o acolhimento, sendo materializado por meio de atitudes evidenciadas nas relações intersubjetivas diariamente estabelecidas entre prestadores e usuários dos serviços. $\mathrm{O}$ acolhimento traduz-se por gestos simples, como forma educada de atendimento, chamar os pacientes pelo nome, informar sobre condutas e procedimentos a serem realizados por intermédio de uma linguagem adequada, escutar e valorizar as narrativas dos usuários, garantir sua privacidade, dentre outras atitudes humanizadoras e éticas ${ }^{13}$. No serviço avaliado a apresentação do protocolo de atendimento ao paciente faz com que ele saiba cada etapa do cuidado em Periodontia e acompanhe seus resultados.

Percebe-se no cenário avaliado que as mulheres procuram atendimento com mais frequência do que os homens, achado que corrobora com outros estudos ${ }^{22,23}$.

A saúde bucal é sócio culturalmente construída como um conceito genérico utilizado para organizar um núcleo de conhecimentos específicos, mas dependente de outros campos de saberes. Portanto, a saúde bucal configura-se como matriz da formação dos cirurgiões-dentistas, mas só se efetiva no encontro com as ações desenvolvidas por diferentes sujeitos, como por exemplo os profissionais da medicina ${ }^{24}$, tendo o sujeito usuário como centro do cuidado sob a influência de determinantes sociais do processo saúde-doença ${ }^{25}$. No presente estudo a maioria dos usuários do serviço apresenta formas mais avançadas de doença periodontal e muitos apresentam envolvimentos sistêmicos associados. Nesse contexto, a disciplina oferece atividades de prevenção e promoção de 
saúde referentes aos principais fatores de risco associados às doenças periodontais.

Para realmente mudar a forma de cuidar, tratar e acompanhar a saúde das pessoas é preciso mudar também o modo de ensinar e aprender, desta forma na Disciplina de Periodontia II da FO/UFRJ o processo de aprendizagem valoriza aspectos relacionados à causa das doenças periodontais e fatores de risco associados. Por exemplo, o estudante é ensinado a regularmente motivar o paciente para a prática de higiene oral, demonstrando técnicas de escovação e uso correto de fio dental/escovas interdentais, assim como a distribuição de folders educativos acerca dos métodos de higiene oral existentes, alimentação correta, efeitos do tabagismo e influência de doenças sistêmicas na saúde bucal ${ }^{21}$.

Há evidências que suportam uma forte ligação entre inflamação, aterosclerose e a Doença Renal Crônica ${ }^{26}$. Biomarcadores inflamatórios como proteína C-reativa e interleucina-6 têm sido encontrados elevados na Doença Renal Crônica. Doenças, como Diabetes mellitus e hipertensão arterial sistêmica, são comumente associadas com Doença Renal Crônica ${ }^{27}$. Esses mesmos marcadores estão aumentados quanto mais grave é a doença periodontal. Desta forma, o enfoque preventivo das atividades extensionistas do serviço colaboram para a prevenção de doenças sistêmicas de grande impacto na qualidade de vida dos indivíduos, bem como na importância do tratamento periodontal na redução desses biomarcadores e melhora em diferentes condições sistêmicas ${ }^{21,28}$.

A identificação dos problemas de saúde apresentados pelos pacientes também é um fator importante, pois possibilita uma ação interprofissional e interdisciplinar na elaboração e execução do plano de tratamento, visando ao atendimento integral do paciente ${ }^{24,29}$.

Ficou demonstrado que os usuários do serviço avaliado apresentam grande percentual de condições sistêmicas associadas, principalmente hipertensão arterial, diabetes mellitus e problemas respiratórios, sendo a abordagem da disciplina voltada para aspectos da Periodontia Médica. Grande parte desse conteúdo é abordado em aulas teóricas e no Projeto de Extensão. A ênfase em uma abordagem interprofissional também é fundamental para que o plano de tratamento do indivíduo seja realizado com base em evidências fundamentadas e com o respaldo de seu médico assistente.

Nos últimos 30 anos a educação interprofissional tem sido inserida nos cursos de saúde com o objetivo de mudar a cultura do atendimento fragmentado dos pacientes. As necessidades atuais dos indivíduos apontam para um atendimento integrado, buscando uma visão holística, sem duplicação de atendimento, diminuindo os erros de diagnóstico, os sobretratamentos e os custos, tanto para o setor público quanto privado. $\mathrm{Na}$ área da saúde, a articulação entre os saberes é fundamental para se aproximar de uma atenção integral à saúde ${ }^{1,24}$.

Uma limitação do estudo foi não estipular o diagnóstico clínico periodontal de maneira completa, visto que nem todos os estudantes classificaram as doenças periodontais com suas subclassificações de distribuição (localizada ou generalizada) e intensidade (leve, moderada ou severa) corretas. Desta forma, os registros de prontuários somente permitiram classificar gengivite ou periodontite, o que aponta necessidade de melhor registro dos diagnósticos realizados nesta clínica.

Outro ponto importante foi a ausência de dados padronizados sobre tabagismo em relação ao tempo de uso e quantidade de cigarros/dia. Estes dados serão inseridos na ficha de anamnese para obtermos futuramente um perfil mais detalhado dos usuários tabagistas. 


\section{CONCLUSÃO}

Foi possível conhecer em detalhes o funcionamento da clínica da disciplina de Periodontia II da FO/UFRJ e os resultados foram utilizados como base para melhorias no serviço. $\mathrm{O}$ perfil de saúde apresentado pelos usuários do serviço apontou para a necessidade de abordagem interprofissional.

\section{ABSTRACT \\ Profile of attendance in the Periodontics service of UFRJ Dental School}

This study aimed to characterize the profile of dental attendance provided by the Periodontics II course offered by the Dental School of Odontology at the Federal University of Rio de Janeiro (FO/UFRJ). We collected ten years' worth of data on dental services (between 2007 and 2016). We analyzed the treatment protocol, the total number of procedures performed per semester and per undergraduate student, the number of periodontal examinations, prophylaxes, oral hygiene instructions, procedures, supragingival scaling procedures, subgingival scaling and root planing performed per semester. Information related to user profiles was collected from the patient's medical chart and included sociodemographic data, periodontal conditions diagnosis, and associated systemic conditions. In the period studied, 13,810 clinical procedures were performed at FO/UFRJ, with an average of 690.5 per semester. In a sample of 715 patients, the age ranged between 12 and 85 years old. The most predominant age range was between 45 and 64 years old, equivalent to $55.38 \%$ of the sample. In the sample, we diagnosed $14.55 \%$ of patients with gingivitis and $85.45 \%$ with periodontitis, of which 93.94\% were chronical and $6.06 \%$ aggressive periodontitis. We identified systemic health conditions associated with periodontal diseases. The most relevant conditions informed by patients were arterial hypertension (25.87\%); respiratory diseases, such as sinusitis and allergic rhinitis (13.29\%); diabetes mellitus (10.35\%), and tobacco use $(7.70 \%)$. We characterized the functioning of the Periodontics II clinic of FO/UFRJ in detail, and our results will be used to improve the service provided for the population in Rio de Janeiro. The profile presented by service users pointed at the need for an interprofessional approach.

Descriptors: Health Services Administration.

Periodontics. Dental education. Interprofessional Care.

\section{REFERÊNCIAS}

1. Lemieux-Charles L, McGuire WL. What do we know about health care team effectiveness? A review of the literature. Med Care Res Rev. 2006; 63:263-300.

2. Brasil. Ministério da Saúde. Secretaria de Atenção à Saúde. Departamento de Atenção Básica. Política Nacional de Atenção Básica / Ministério da Saúde. Secretaria de Atenção à Saúde. Departamento de Atenção Básica. Brasília: Ministério da Saúde, 2012.

3. Kusma SZ, Moysés ST, Moysés SJ. Promoção da Saúde: perspectivas avaliativas para a saúde bucal na atenção primária em saúde. Cad Saúde Pública. 2012; 28:s9-s19.

4. Donabedian A. The seven pillars of quality. Arch. Pathol Lab Med 1990; 114:1115-18.

5. Righi AW. Avaliação da qualidade em serviços públicos de saúde - o caso da Estratégia Saúde da Família. [dissertação]. Santa Maria: UFSM; 2009.

6. Arouca R. Breve histórico ilustrado da Faculdade de Odontologia da UFRJ. Rio de Janeiro: Editora Santos; 2009.

7. Conselho Nacional de Educação/ Câmara de Educação Superior (Brasil). Resolução $\mathrm{n}^{\circ} 3$ de 19 de fevereiro de 2002. Diretrizes Curriculares Nacionais do Curso de 
Graduação em Odontologia. Diário Oficial da União, Brasília, 4 de março de 2002. Seção 1, p. 10.

8. Secretaria Municipal de Saúde. Plano Municipal de Saúde do Rio de Janeiro 20142017.

9. Cugini MA, Haffajee AD, Smith C, Kent Jr RL, Socransky SS. The effect of scaling and root planing on the clinical and microbiological parameters of periodontal diseases: 12-month results. J Clin Periodontol. 2000; 27: 30-6.

10. Gomes-da-Silva D, Tosto MC, Evangelista MT, Silva JNO, Abreu T, Monteiro CLS, et al. Protocolo de atendimento da Clínica de Periodontia Unigranrio: pacientes com diabetes mellitus. Rede de Cuidados em Saúde 2017; 10:1-12.

11. Secretaria de Estado de Saúde - Subsecretaria de Atenção Integral à Saúde (Distrito Federal). Portaria $\mathrm{n}^{\circ} 342$ de 28 de junho de 2017. Institui protocolo de atenção à saúde- Periodontia. Diário Oficial do DF 2017; 30 de jun.

12. Armitage GC. Development of a classification system for periodontal disease and conditions. Ann Periodontol. 1999; 4(1):1-6.

13. Brasil. Ministério da Saúde. Política Nacional de Humanização. Brasília, 2004.

14. Merhy EE. Em busca do tempo perdido: a micropolítica do trabalho vivo em saúde. In: Merhy EE, Onocko R. Agir em saúde um desafio para o público. São Paulo: HUCITEC, 1997. p. 71-112.

15. Merhy EE. Saúde: a cartografia do trabalho vivo. $3^{\text {a }}$ ed. São Paulo: Hucitec; 2002.

16. Campos GWS. Um método para análise e cogestão de coletivos. São Paulo: HUCITEC; 2000 .
17. Campos GWS. Saúde Paidéia. São Paulo: HUCITEC; 2003.

18. Gaunt F, Devine M, Pennington M, Vernazza C, Gwynnett E, Steen N, Heasman P. The cost effectiveness of supportive periodontal care for patients with chronic periodontitis. J Clin Periodontol. 2008; 35: 67-82.

19. Tonetti MS, Eickholz P, Loos BG, Papapanou P. Principles in prevention of periodontal diseases-Consensus report of group 1 of the 11th European workshop on periodontology on effective prevention of periodontal and peri-implant diseases. J Clin Periodontol. 2015; 42 (16): S5-S11.

20. Pretzl B, El Sayed S, Weber D, Eickholz P, Bäumer A. Tooth loss in periodontally compromised patients: results 20 years after active periodontal therapy. J Clin Periodontol. 2018 45:1356-64.

21. Sabharwal A, Gomes-Filho IS, Stellrecht E, Scannapieco FA. Role of periodontal therapy in management of common complex systemic diseases and conditions: an update. Periodontol 2000. 2018;78(1):212-26.

22. Pinheiro RS, Viacava F, Travassos C, Brito AS. Gênero, morbidade, acesso e utilização de serviços de saúde no Brasil. Ciên Saude Colet 2002; 7(4):687-707.

23. Pandolfi M, Barcellos LAE, Miotto MHMB. Perfil dos usuários e motivo da procura pelos serviços odontológicos das unidades de saúde de Vitória - ES. UFES Rev Odontol. 2006; 8(2):37-44.

24. Reeves S, Xyrichis A, Zwarenstein M. Teamwork, collaboration, coordination, and networking: why we need to distinguish between different types of interprofessional practice. J Interprof Care. 2018; 32(1):1-3.

25. Vettore MV, Marques RADA, Peres MA. 
2013. Desigualdades sociais e doença periodontal no estudo SB Brasil 2010: abordagem multinível. Rev Saúde Públ 2010; 47: 29-39.

26. Schiffrin EL, Lipman ML, Mann JFE. Chronic Kidney Disease: Effects on the cardiovascular system. Circulation. 2007;3:85-97.

27. Scannapieco FAE, Panesar M. Periodontitis and chronic kidney disease. J Periodontol. 2008; 79:1617-9.

28. Artese HPC, Sousa CO, Luiz RR, Sansone C, Torres MCMB. Effect of non-surgical periodontal treatment on chronic kidney disease patients. Braz Oral Res. 2010;
24(4):449-54.

29. Ponzer S, Hylin U, Kusoffsky A, Lauffs M, Lonka K, Mattiasson A-C, Nordström G. Interprofessional training in the context of clinical practice: goals and student's perception on clinical education wards. Med Educ. 2004; 38:727-36.

\section{Correspondência para:}

Cláudia Callegaro de Menezes

e-mail: cacamenezes@ hotmail.com

Rua Professor Rodolpho Paulo Rocco, 325

Ilha do Fundão

CEP 21941-617 Rio de Janeiro/RJ 